\title{
Phosphoproteomic analysis of the non-seed vascular plant model Selaginella moellendorffii
}

\author{
Xi Chen ${ }^{1,2 \dagger}$, Wai Lung Chan ${ }^{1 \dagger}$, Fu-Yuan Zhu ${ }^{1}$ and Clive Lo ${ }^{1 *}$
}

\begin{abstract}
Background: Selaginella (Selaginella moellendorffii) is a lycophyte which diverged from other vascular plants approximately 410 million years ago. As the first reported non-seed vascular plant genome, Selaginella genome data allow comparative analysis of genetic changes that may be associated with land plant evolution. Proteomics investigations on this lycophyte model have not been extensively reported. Phosphorylation represents the most common post-translational modifications and it is a ubiquitous regulatory mechanism controlling the functional expression of proteins inside living organisms.

Results: In this study, polyethylene glycol fractionation and immobilized metal ion affinity chromatography were employed to isolate phosphopeptides from wild-growing Selaginella. Using liquid chromatography-tandem mass spectrometry analysis, 1593 unique phosphopeptides spanning 1104 non-redundant phosphosites with confirmed localization on 716 phosphoproteins were identified. Analysis of the Selaginella dataset revealed features that are consistent with other plant phosphoproteomes, such as the relative proportions of phosphorylated Ser, Thr, and Tyr residues, the highest occurrence of phosphosites in the C-terminal regions of proteins, and the localization of phosphorylation events outside protein domains. In addition, a total of 97 highly conserved phosphosites in evolutionary conserved proteins were identified, indicating the conservation of phosphorylation-dependent regulatory mechanisms in phylogenetically distinct plant species. On the other hand, close examination of proteins involved in photosynthesis revealed phosphorylation events which may be unique to Selaginella evolution. Furthermore, phosphorylation motif analyses identified Pro-directed, acidic, and basic signatures which are recognized by typical protein kinases in plants. A group of Selaginella-specific phosphoproteins were found to be enriched in the Pro-directed motif class.

Conclusions: Our work provides the first large-scale atlas of phosphoproteins in Selaginella which occupies a unique position in the evolution of terrestrial plants. Future research into the functional roles of Selaginella-specific phosphorylation events in photosynthesis and other processes may offer insight into the molecular mechanisms leading to the distinct evolution of lycophytes.
\end{abstract}

Keywords: Selaginella, IMAC, PEG fractionation, Phosphosites, Phosphorylation motif

\section{Background}

Selaginella (Selaginella moellendorffii) is a lycophyte believed to be originated from the earliest vascular plants approximately 410 million years ago [1]. Although lycophytes have existed twice as long as angiosperms, they have not evolved flowers and seeds since their divergence from other plant lineages. For this reason, Selaginella has

\footnotetext{
*Correspondence: clivelo@hku.hk

†Equal contributors

'School of Biological Sciences, The University of Hong Kong, Pokfulam

Hong Kong, China

Full list of author information is available at the end of the article
}

been selected as a model plant to understand the early evolution of developmental and metabolic processes that are unique to vascular plants [2]. After a bacterial artificial chromosome library was constructed from clonally propagated plants [3], the complete Selaginella genome sequence was released in 2007 [4]. Subsequently, a number of investigations on Selaginella were launched in different areas including gene evolution [5-10], pathway conservation [11-16], genomic DNA composition and methylation [17-19], sRNA functions and RNA editing [20,21], and transposons [22]. Interestingly, Selaginella was found to utilize genes significantly different from flowering plants 
to generate secondary metabolites with potentials for pharmaceutical applications [23-26]. Meanwhile, proteomic investigations on this non-seed vascular plant model have not been extensively reported. A two-dimensional electrophoresis-based approach was recently employed to explore the desiccation tolerance mechanism in the resurrection plant Selaginella tamariscina [27].

Post-translational modifications (PTMs) play important roles in the regulation of protein functions and they occur at distinct amino acid side chains or peptide linkages. It has been estimated that more than 200 types of PTMs exist in proteins [28]. Protein phosphorylation, principally on serine, threonine or tyrosine residues, is one of the most important and well-investigated PTMs. It represents a reversible molecular switch controlled by protein kinases and protein phosphatases, either activating or inactivating the target proteins [29]. Approximately one-third of all proteins in eukaryotic cells were estimated to be phosphorylated at any given time [30]. In plants, protein phosphorylation plays a central role in virtually all cellular processes, including carbon and nitrogen metabolism, growth and development, transcription and translation, responses to abiotic and biotic stresses, cell cycle, and apoptosis [31]. Therefore, the identification of protein kinases and phosphatases, their substrates, and the phosphorylation sites involved is crucial for the understanding of many fundamental processes in plants. Interestingly, Arabidopsis contains over 1000 protein kinases [32], which is twice as many as those in human, while the two genomes share similar number of genes [33]. Hence, protein phosphorylation events in plants appear to be very different and more complicated than those in mammals. In fact, a number of plant protein kinases implicated in early events of signal transduction are unique with no mammalian orthologs [34].

Phosphoproteomic investigations in plants were initiated in recent years following the completion of different genome sequencing projects. The highly abundant ribulose-1,5-bisphosphate carboxylase/oxygenase (RUBISCO) protein, which accounts of about $50 \%$ of total soluble proteins [35], hindered the detection of low-abundant proteins including many phosphoproteins [36]. Polyethylene glycol (PEG) fractionation has been used as a costeffective and contaminant-free procedure to remove RUBISCO for improved detection of low-abundant proteins [37-39]. In addition, phosphopeptide enrichment procedures, such as immobilized metal ion affinity chromatography (IMAC), are necessary to reduce the complexity of proteolyzed lysates for mass spectrometry analysis. IMAC is based on affinity purification through metal complexation with the phosphate group in phosphopeptides [40] and it has been adopted in Phosphoproteomic analysis in different plant systems [41-46].
In the present study, we used the PEG fractionation approach followed by the IMAC procedure to prepare Selaginella samples for phosphoproteome profiling and identified 1588 unique phosphorylation sites. Our dataset revealed features that are consistent with the Arabidopsis phosphoproteome. We further identified phosphorylation events that are conserved between Selaginella and angiosperm orthologous sequences. Novel and unique phosphosites were detected in several photosynthesis-related proteins in Selaginella. Phosphorylation motifs recognized by known protein kinase classes were revealed for both evolutionarily conserved and Selaginella-specific proteins.

\section{Results and discussion}

\section{General features of the Selaginella phosphoproteome dataset}

We employed the procedures of PEG fractionation (Additional file 1: Figure S1) and IMAC enrichment to isolate phosphopeptides from wild-growing Selaginella (Selaginella moellendorffii) for LC-MS/MS analysis. A total of 1593 unique phosphopeptides containing 1588 non-redundant phosphosites were discovered in our study (Additional file 2: Table S1). Among them, 1104 were identified with high confidence of localization (localization probability $\geq 95 \%$ ), 116 with median confidence of localization $(80 \% \leq$ localization probability $<95 \%)$, and 368 with low confidence of localization (localization probability < 80\%). Phosphosites with high confidence of localization were categorized into pSer (86.2\%), pThr (13.3\%), and pTyr (0.5\%). The relative distribution of the three phosphorylated residues is consistent with previous reports for different flowering plant species [45,46]. As Ser/Thr kinases are commonly encoded in plant genomes, more frequent Ser and Thr phosphorylation events are expected. On the other hand, while typical Tyr-specific kinases are absent in plant genomes, a few plant kinases with dual specificity are believed to phosphorylate Tyr residues in proteins [47].

The 1104 confirmed phosphosites correspond to a total of 716 Selaginella proteins, 665 of them can be assigned to orthologous protein groups (Additional file 3: Table S2) using the OrthoMCL algorithm with a cut-off of E-5 e-value and $50 \%$ sequence match $[48,49]$. Forty two proteins are considered Selaginella-specific proteins since they could not be assigned to any OrthoMCL groups or do not have any matching sequences in the OrthoMCL database. These proteins may have evolved in lycophytes after their separation from other vascular plants including ferns and seed plants.

\section{Analysis of phosphosite locations in Selaginella proteins}

To analyze the locations of the identified phosphorylation sites, protein sequences were divided into $5 \%$ fractions and the number of phosphorylation events was counted 
within each fraction. As shown in Figure 1A, the highest number of phosphosites is found in the last fraction, i.e. the C-termini of proteins. We performed parallel analysis using an Arabidopsis phosphoproteome dataset (retrieved from $\mathrm{P}^{3} \mathrm{DB}$ ) and found a very similar distribution pattern for the phosphosites (Figure 1B). Such phenomenon was also described in a phosphoproteomic study of mouse liver [50]. Hence, the more frequent C-terminal phosphorylation in proteins appears to be a common feature in different organisms, including plants and animals. The C-terminal region was suggested to be more exposed and flexible for protein phosphorylation [50].

\section{Functional categorization of the identified Selaginella phosphoproteins}

To understand the functional distribution of the unique Selaginella phosphoproteins identified in this study, their cellular localization, molecular function, and biological processes were analyzed and compared with those of 2400 Selaginella proteins identified after LC-MS/MS analysis of PEG-fractionated samples without the IMAC enrichment procedure. Based on the comparison of Gene Ontology (GO) term annotations (Figure 2), the 3 most overrepresented categories for the identified phosphoproteins in each GO vocabulary are: nucleus, plasma membrane and cytosol for "cellular component"; DNA/RNA binding,

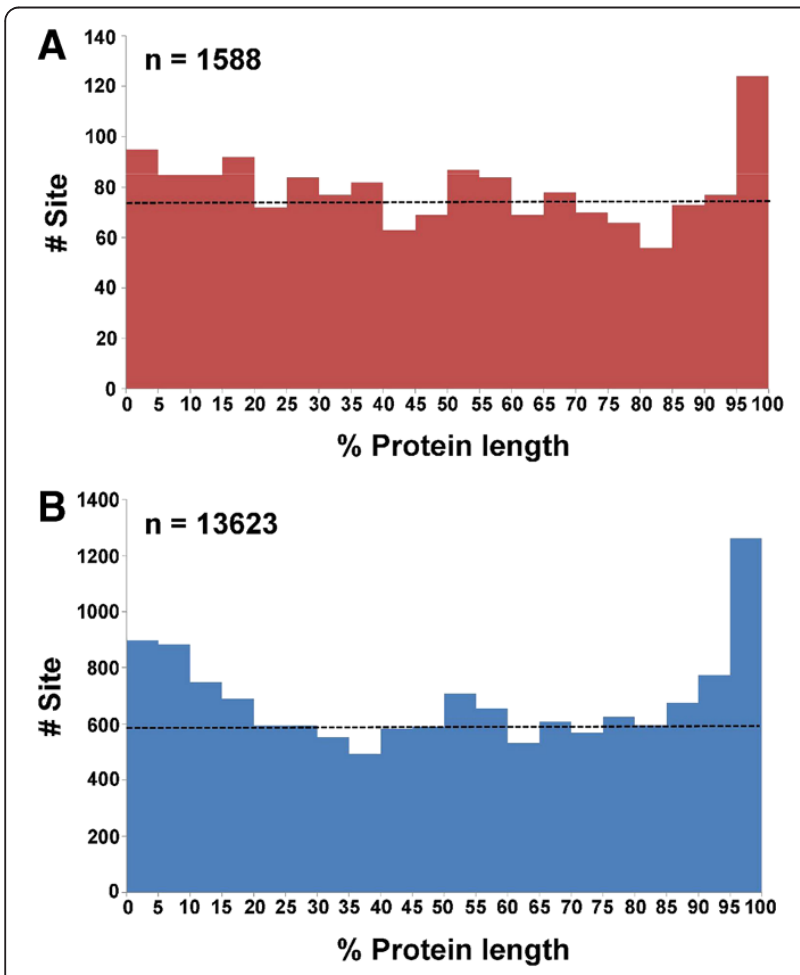

Figure 1 The distribution of phosphorylation sites along the phosphoproteins of (A) Selaginella and (B) Arabidopsis. The Arabidopsis dataset was retrieved from $P^{3} D B$.

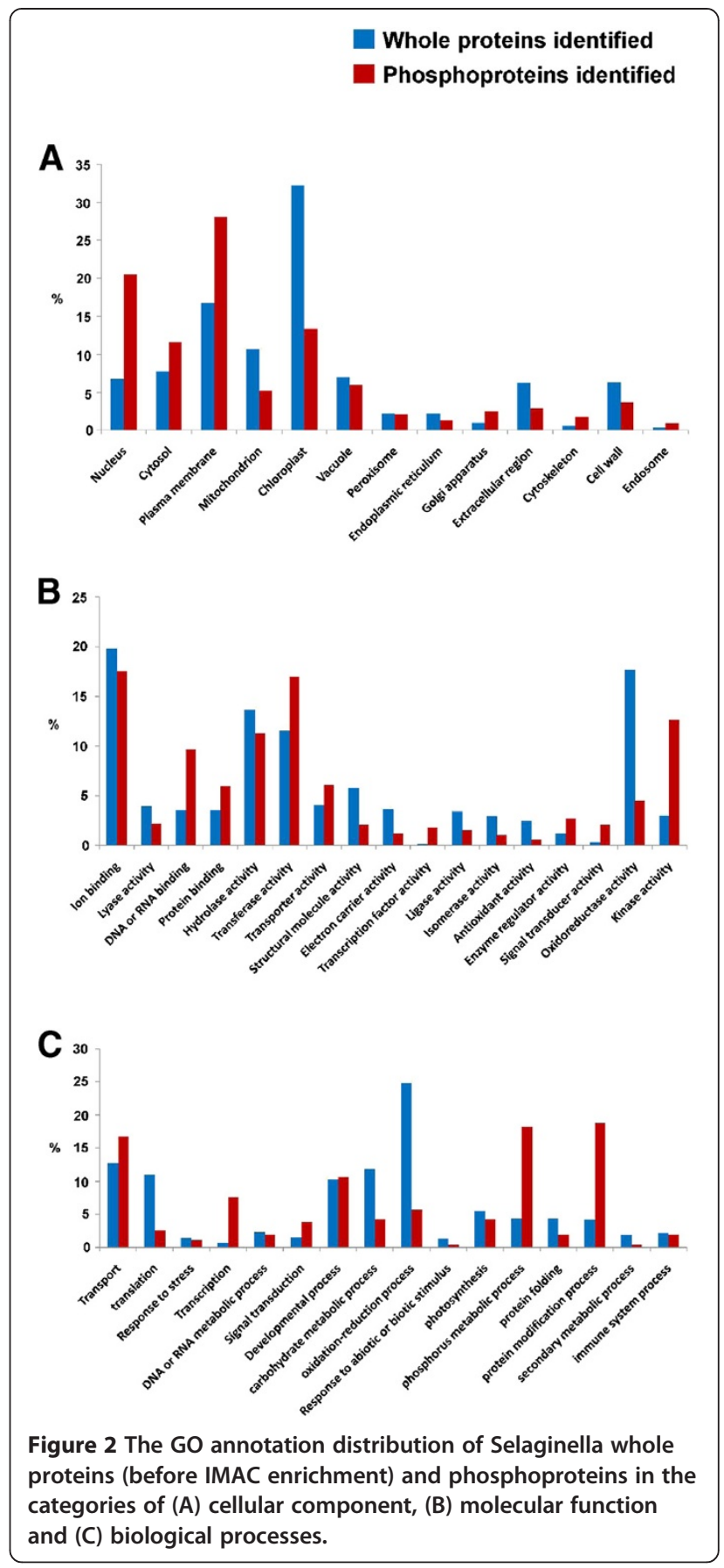

kinase activity, and transferase activity for "molecular function"; protein modification, phosphorus metabolic process, and transcription for "biological process".

\section{Location of phosphosites in characterized protein domains}

To determine whether the Selaginella phosphosites are located in known structural and/or functional protein domains, Pfam database search (Wellcome Trust Sanger Institute) was performed to extract domain information 
of our identified phosphoproteins. A total of 594 proteins with domain information were retrieved. Among the 893 phosphosites in these proteins, only 201 (22.5\%) were located inside protein domains (Table 1). Our findings are consistent with the observations from Arabidopsis phosphoproteome analysis suggesting that phosphorylation events may not have significant impact on domain-associated functions [51,52].

\section{Phosphorylation motif analysis}

A phosphorylation motif search was performed on our phosphopeptide dataset (localization probability $\geq 95 \%$ ) using the Motif-X algorithm [53]. Peptide sequences are aligned with their length adjusted to \pm 7 residues from the central phosphosite for data submission. Over-represented patterns of amino acid sequences were generated with a minimum occurrence of 20 and a significance value of $10^{-6}$. All together, we obtained a total of 11phosphorylation (9 Ser and $2 \mathrm{Thr}$ ) motifs containing at least one fixed amino acid aside from the central phosphorylated residue (Figure 3A). Both the Thr-motifs are Pro-targeted (TP and PXTP) and there are 3 Ser Pro-targeted motifs (SP, PXSP, SPXR). All these motifs are possible substrates of glycogen synthase kinase 3, cyclin-dependent kinase, and mitogen-activated protein kinase. In addition, 3 basophilic motifs (LXRXXS, RXXS, KXXS) likely to be associated with the activities of $\mathrm{Ca}^{2+}$-dependent protein kinase (CPK), $\mathrm{Ca}^{2+} /$ calmodulin-dependent protein kinase, or protein kinase A were identified. Furthermore, 3 acidic motifs (SDXE, SXD, and SE) potentially recognized by casein kinase II were generated. We also performed parallel Motif-X analysis using Arabidopsis phosphopeptides retrieved from $\mathrm{P}^{3} \mathrm{DB}$ and those obtained by Wang et al. (2013). One of the Selaginella motifs, KXXS, was not generated from the Arabidopsis analysis. Thirty two (out of 38) occurrences of such motif correspond to proteins assigned with OrthoMCL group with e-value $<\mathrm{E}^{-50}$ (Additional file 4: Table S3), indicating that this basophilic motif is primarily associated with evolutionarily conserved proteins in Selaginella. Analysis of the 107 phosphosites in the Selaginella-specific proteins

Table 1 Location of phosphorylation sites on characterized protein domains

\begin{tabular}{|c|c|c|c|c|}
\hline \multirow{3}{*}{\multicolumn{2}{|c|}{$\begin{array}{l}\text { Number of } \\
\text { proteins } \\
\text { possessing } \\
\text { Pfam domain }\end{array}$}} & \multicolumn{3}{|c|}{ Number of phosphorylation sites ${ }^{(1)}$} \\
\hline & & \multicolumn{2}{|c|}{ Pfam domain $^{(2)}$} & \multirow[t]{2}{*}{ Total (\%) } \\
\hline & & \multirow{2}{*}{$\begin{array}{l}\text { Inside (\%) } \\
163(21.0 \%)\end{array}$} & \multirow{2}{*}{$\begin{array}{l}\text { Outside (\%) } \\
612(79.0 \%)\end{array}$} & \\
\hline pS & 538 & & & 775 (100\%) \\
\hline pT & 102 & 36 (31.6\%) & 78 (68.4\%) & $114(100 \%)$ \\
\hline pY & 4 & $2(50 \%)$ & $2(50 \%)$ & $4(100 \%)$ \\
\hline All & 594 & $201(22.5 \%)$ & 692 (77.5\%) & $893(100 \%)$ \\
\hline
\end{tabular}

${ }^{(1)}$ All analyzed phosphorylation sites were confirmed with the localization probability above $95 \%$ deduced from Ascore.

${ }^{(2)}$ Location of phosphorylation sites relative to the conserved domains annotated in the Pfam database. (those without any assigned OrthoMCL groups) revealed that they are more enriched in Pro-directed motifs when compared to all the identified phosphosites (49\% vs 35\%) (Figure 3B). On the other hand, the basophilic motifs are under-represented in the Selaginella-specific proteins when compared to all proteins identified (23\% vs $38 \%$ ). Consistently, a single SP motif with 36 occurrences was generated by Motif-X analysis for the 107 phosphosites found in the Selaginella-specific proteins. Taken together, most of the Selaginella phosphorylation events identified in this study are likely to be catalyzed by known classes of protein kinase classes in plants.

\section{Phosphorylation events in evolutionary conserved proteins}

To identify phosphorylation events highly conserved between Selaginella and flowering plants, our identified phosphopeptides with confirmed phosphosites were clustered with phosphopeptides of Arabidopsis, rice, rapeseed, soybean and Medicago truncatula (retrieved from $\mathrm{P}^{3} \mathrm{DB}$ ) by CD-HIT [54] using a sequence identity cutoff of 0.6 and an alignment bandwidth of 5 [44]. A total of 107 Selaginella phosphopeptides harboring 115 Ser/Thr phosphosites were found to cluster with phosphopeptides from the other plants. More than 80\% (97/123) of those Selaginella phosphosites were found to have equivalent phosphosites in at least one other species. The majority (90/106) of the Selaginella proteins harboring the conserved phosphosites are evolutionarily conserved proteins belonging to Orthogroups identified with e values $<$ E-50 (Additional file 5: Table S4). Many of these proteins are involved in primary metabolism (e.g. Calvin cycle, glycolysis, TCA cycle, lipid biosynthesis), RNA processing, transcriptional regulation, cell cycle, protein phosphorylation (kinases), and signaling (e.g. G proteins, 14-3-3 protein, LRR-containing kinases). On the other hand, 17 of these highly conserved phosphorylation events are found in proteins with unknown functions. Selected phosphopeptide alignments containing highly conserved phosphosites in multiple plant species are shown in Table 2.

Furthermore, we performed a close examination on the phosphorylation events in Selaginella photosynthesisrelated proteins. The molecular machinery of photosynthesis has been highly conserved during plant evolution. Among our identified phosphoproteins with confirmed phosphosites, seven are involved in photosystem II (PSII) and two are involved in photosystem I (PSI) (Figure 4A). To reveal possible evolutionary significance, sequences were aligned with orthologs from Arabidopsis, rice, and Physcomitrella patens (moss), representing diverse lineages of dicot, monocot, and bryophytes, respectively (Figure 4B and Additional file 6: Figure S2). In all cases, phosphorylation information is only available for the Arabidopsis proteins. Sequences of rice and moss are 


\begin{tabular}{|c|c|c|c|}
\hline $\begin{array}{l}\text { Serine/Threonine } \\
\text { Motifs }^{(1)}\end{array}$ & $\begin{array}{c}\text { No. of } \\
\text { Occurences }\end{array}$ & Kinase Reported & Motifs Reported \\
\hline$\ldots \ldots$ P.sp..... & 79 & $\begin{array}{l}\text { Extracellular signal-regulated kinase, } \\
\text { MAPK }\end{array}$ & A.K. Pullikuth $e t a l .{ }^{(2)}$ \\
\hline$\ldots \ldots$ sP.R... & 53 & $\begin{array}{l}\text { Growth associated histone HI kinase, } \\
\text { CDK1, } 2,4,6, \text { CDC2 }\end{array}$ & A. Errico et al. ${ }^{(3)}$ \\
\hline$\ldots \ldots$ sp $\ldots \ldots$ & 280 & Proline-directed kinase, MAPK, CDC2 & $\mathrm{P} 3 \mathrm{DB}^{(4)} / \mathrm{X}$. Wang et al. ${ }^{(5)}$ \\
\hline .L.R.S...... & 51 & OST1 & P3DB \\
\hline 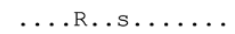 & 111 & SPK, CDPK & P3DB / X. Wang et al. \\
\hline .....sD.E... & 27 & Casein kinase II & P3DB / X. Wang et al. \\
\hline ............. & 50 & Casein kinase II & P3DB \\
\hline$\ldots \ldots$ sE.... & 37 & & $\mathrm{X}$. Wang et al. \\
\hline ...к. & 38 & & \\
\hline$\ldots \ldots$ P.tp $\ldots \ldots$ & 26 & MAPK, GSK3, CDK5 & $\mathrm{X}$. Wang et al. \\
\hline$\ldots \ldots$ tp $\ldots \cdots$ & 55 & Pro-directed kinase & $\mathrm{P} 3 \mathrm{DB} / \mathrm{X}$. Wang et al. \\
\hline
\end{tabular}

(1) Phosphorylation of serine or threonine as indicated as 's' or 't'.

(2) A.K. Pullikuth et al, Mol. Cell Biol. 30: 3233-3248.

(3) A. Errico et al., Advances in Enzyme Regulation 50: 375-399.

(4) Non-redundant phosphopeptides provided from Plant Protein Phosphorylation Database (P3DB).

(5) Phosphopeptides data obatined from X. Wang et al., J. of Proteomics 78: 486-498.

\section{B}

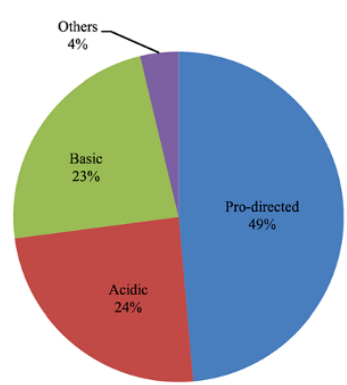

(I) P-sites in S. moellendorffii unique protein

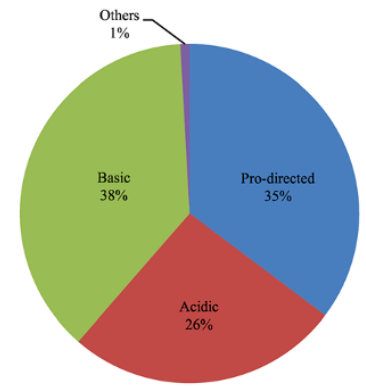

(II) P-site in all protein

Figure 3 Phosphorylation motifs in Sellaginella phosphoproteins. (A) Motif-x analysis of phosphorylation peptides with localization probability 95\%. (B) Motif classes distribution of Selaginella(Sm) specific phosphorylation site (P-site).

included for examination of phosphorylatable residues at equivalent sites.

As the first link in the chain of light-dependent reactions, PSII captures photons and uses the energy to extract electrons from water molecules. The light-harvesting chlorophyll a/b protein complex LHCII in PSII is composed of three proteins, namely Lhcb1, Lhcb2, and Lhcb3 [55]. Non-phosphorylated LHCII functions as an antenna for PSII, but it migrates to come in contact with PSI following light-dependent phosphorylation of Lhcb1 and Lhcb2 [56] which is likely to occur at N-terminal Thr residues [57]. Although no explicit phosphosites were identified [55-57], STN7 kinase was strongly suggested to be required for phosphorylation of Lhcb1 and Lhcb2 to achieve state transitions between PSII and PSI [56]. In Selaginella, we detected N-terminal Thr phosphosites in D8QN27 (Lhcb1: Thr-44) and D8SUF1 (Lhcb2: Thr-42) (Figure 4B). Both phosphorylation events are conserved in Arabidopsis Lhcb1 and Lhcb2 [58,59]. Equivalent phosphorylatable residues are also found in rice Lhcb1 and Lhcb2 (Figure 4B). As these Thr residues are preceded by a basic residue (Lys or Arg), they represent potential signatures recognized by STN7 [60]. In fact, the Thr-40 in Arabidopsis Lhcb2is phosphorylated in wild-type but not in the $\sin 7$ mutant [59], further suggesting that it is a target of STN7. In D8QN27 (Lhcb1), we also identified the Ser-54 Pro-directed phosphosite which is conserved in Arabidopsis Lhcb1 [43] and an equivalent Ser residue in rice Lhcb1 (Figure 4B). Interestingly, all the $\mathrm{N}$-terminal Ser/Thr residues mentioned above are not conserved in $P$. patens Lhcb1 and Lhcb2 and they were probably only evolved after the emergency of vascular plants. On the other hand, the Ser- 48 and Ser- 49 phosphosites in D8QN27 (Lhcb1) are located in a region not conserved with the Arabidopsis and rice sequences, but equivalent Ser residues are identified in moss Lhcb1. They may represent phosphorylation events that are lost in the angiosperm lineage.

Lhcb4, a minor chlorophyll-binding protein, was found to be phosphorylated in maize upon exposure to high light intensity for protection against cold stress [61]. The phosphosite Thr-112, a potential casein kinase II target, 
Table 2 Selected conserved phosphorylation sites within the orthologous proteins

\begin{tabular}{|c|c|c|c|c|c|}
\hline Accession & Phosphopeptides alignment $^{(1)}$ & Protein annotation & MapmanBin name & OrthoMCL group & E-value \\
\hline D8QZX4 & WLEGGsDDEGASTEAHGR & \multirow[t]{3}{*}{ ILITYHIA } & \multirow[t]{3}{*}{ Not assigned.no ontology } & \multirow[t]{3}{*}{ OG5_128125 } & \multirow[t]{3}{*}{$1 \mathrm{E}-181$} \\
\hline AT1G64790.1 & ALLEGGsDDEGASTEAQGR & & & & \\
\hline Os03g51140.1 & AlLEGGsDDEGASTEAHGR & & & & \\
\hline D8RKK4 & SNsFVGTEEYIAPEIIK & \multirow[t]{3}{*}{ KCBP-interacting protein kinase } & \multirow[t]{3}{*}{ Protein. stranslational modification } & \multirow[t]{3}{*}{ OG5_129514 } & \multirow[t]{3}{*}{$1 \mathrm{E}-181$} \\
\hline AT3G52890.2 & SNsFVGTHEYLAPEIIK & & & & \\
\hline Os02g43740.1 & SMsFVGTHEYLAPEIIK & & & & \\
\hline D8T2E8 & ASGAFILTAsHNPGGPHEDFGIK & \multirow{3}{*}{$\begin{array}{l}\text { Phosphoglucomutase/phosphomannomutase } \\
\text { family protein }\end{array}$} & \multirow[t]{3}{*}{ Glycolysis.PGM } & \multirow[t]{3}{*}{ OG5_127226 } & \multirow[t]{3}{*}{$1 E-181$} \\
\hline AT1G23190.1 & ATGAFILTAsHNPGGPTEDFGIK & & & & \\
\hline Os03g50480.1 & ATGAFILTAsHNPGGPTEDFGIK & & & & \\
\hline D8SQG3 & sQAGTPEWMAPEVLR & \multirow[t]{3}{*}{ Protein kinase superfamily protein } & \multirow[t]{3}{*}{ Signalling.MAP kinases } & \multirow[t]{3}{*}{ OG5_130251 } & \multirow[t]{3}{*}{$1 \mathrm{E}-181$} \\
\hline AT1G08720.1 & sTAGTPEWMAPEVLR & & & & \\
\hline Os02g50970.2 & STAGTAEWMAPEVLR & & & & \\
\hline D8SCJ0 & ALVANYNQtPR & \multirow[t]{3}{*}{ Cell division cycle 5} & \multirow{3}{*}{$\begin{array}{l}\text { RNA.regulation of transcription.MYB-related } \\
\text { transcription factor family }\end{array}$} & \multirow[t]{3}{*}{ OG5_128000 } & \multirow[t]{3}{*}{$1 \mathrm{E}-181$} \\
\hline AT1G09770.1 & ALLANYSQtPR & & & & \\
\hline Os04g28090.1 & ALLSSYSQtPR & & & & \\
\hline D8RHD1 & GILAMDESNATCGK & \multirow[t]{3}{*}{ Aldolase superfamily protein } & \multirow[t]{3}{*}{ Glycolysis.aldolase } & \multirow[t]{3}{*}{ OG5_127143 } & \multirow[t]{3}{*}{$1 \mathrm{E}-179$} \\
\hline AT3G52930.1 & GILAADESTGTIGK & & & & \\
\hline Os05g33380.1 & GILAADEsTGTIGK & & & & \\
\hline D8R365 & GFVPILPGsPGSS & \multirow[t]{3}{*}{$\mathrm{Na}+/ \mathrm{H}+$ exchanger 1} & \multirow[t]{3}{*}{ Transport.unspecified cations } & \multirow[t]{3}{*}{ OG5_126729 } & \multirow[t]{3}{*}{$1 E-181$} \\
\hline AT5G27150.1 & GFVPFVPGSPTER & & & & \\
\hline Os1 1g42790.1 & GFVPFVPGsPTER & & & & \\
\hline $\mathrm{D} 8 \mathrm{Q} \times 62$ & PsGSPPVPVMHsPPRPVTVK & \multirow[t]{3}{*}{ Chromatin protein family } & DNA.synthesis/chromatin structure & OG5_128357 & $1 \mathrm{E}-181$ \\
\hline AT1G77180.1 & AsGSPPVPVMHsPPRPVTVK & & & & \\
\hline Os02g52250.1 & AsGSPPVPVMHsPPRPVTVK & & & & \\
\hline D8QU29 & YHGHsMSDPGSTYR & Pyruvate dehydrogenase complex E1 & TCA/org.transformation.TCA. & OG5_127216 & $1 \mathrm{E}-176$ \\
\hline AT1G59900.1 & YHGHsMSDPGSTYR & alpha subunit & Pyruvate DH.EI & & \\
\hline Medtr5g037700.1 & YHGHsMSDPGSTYR & & & & \\
\hline D8SRX1 & WGTQAPVQLGSLR & GDP-D-mannose 3',5'-epimerase & Redox. ascorbate and glutathione. & OG5_135736 & $1 \mathrm{E}-181$ \\
\hline AT5G28840.1 & WGTQAPVQLGsLR & & & & \\
\hline Os10g28200.1 & WSTQAPVQLGSLR & & & & \\
\hline D8RPK5 & AHGPAVGLPTEDDMGNsEVGHNALGAGR & Phosphoglycerate mutase, & Glycolysis.phosphoglycerate mutase & OG5_129051 & $1 \mathrm{E}-181$ \\
\hline AT1G09780.1 & AHGTAVGLPSEDDMGNsEVGHNALGAGR & 2,3-bisphosphoglycerate-Independent & & & \\
\hline Os05g40420.3 & AHGTAVGLPSDDDMGNsEVGHNALGAGR & & & & \\
\hline
\end{tabular}


Table 2 Selected conserved phosphorylation sites within the orthologous proteins (Continued)

\begin{tabular}{|c|c|c|c|c|c|}
\hline D8SC69 & VQSSSAIWHPR & \multirow{2}{*}{$\begin{array}{l}\text { Receptor protein kinase TMK1 precursor, putative, } \\
\text { expressed }\end{array}$} & \multirow[t]{3}{*}{-} & \multirow[t]{2}{*}{ OG5_141123 } & \multirow[t]{2}{*}{$1 \mathrm{E}-181$} \\
\hline Os03g50810.1 & VQSPHAMWHPR & & & & \\
\hline Glyma02g40980.1 & VQsPNALVIHPR & & & & \\
\hline D8SOV4 & QLsIDQFENEGR & \multirow[t]{3}{*}{ BRI1 suppressor 1 (BSU1)-like 2} & \multirow[t]{3}{*}{ Protein.postranslational modification } & \multirow[t]{3}{*}{ OG5_132764 } & \multirow[t]{3}{*}{$1 \mathrm{E}-181$} \\
\hline AT1G08420.1 & QLSIDQFENEGR & & & & \\
\hline Os12g42310.1 & QLSIDQFENEGR & & & & \\
\hline D8QXA8 & NFRPDsLLGEGGFGSVFK & \multirow[t]{3}{*}{ Protein kinase superfamily protein } & \multirow{3}{*}{$\begin{array}{l}\text { Protein.postranslational modification. } \\
\text { kinase.receptor like cytoplasmatic kinase VII }\end{array}$} & \multirow{3}{*}{ OG5_147118 } & \multirow[t]{3}{*}{$1 \mathrm{E}-163$} \\
\hline AT1G07570.1 & NFRPDsVLGEGGFGCVFK & & & & \\
\hline Glyma01g05160.1 & NFRPDsLLGEGGFGYYK & & & & \\
\hline D8RMG5 & ALsPDRNDAFAMGDK & \multirow[t]{3}{*}{ Splicing factor, putative } & \multirow[t]{3}{*}{ RNA.processing.splicing } & \multirow[t]{3}{*}{ OG5_127822 } & \multirow[t]{3}{*}{$1 \mathrm{E}-181$} \\
\hline AT5G64270.1 & VLSPDRVDAFAMGDK & & & & \\
\hline Medtr2g009180.1 & ILSPDRHDAFAAGEK & & & & \\
\hline D8RZ45 & LTSFEALQSATK & \multirow[t]{3}{*}{ ADP-glucose pyrophosphorylase family protein } & \multirow{3}{*}{$\begin{array}{l}\text { Major CHO metabolism.synthesis. } \\
\text { starch.AGPase }\end{array}$} & \multirow[t]{3}{*}{ OG5_129964 } & \multirow[t]{3}{*}{$1 \mathrm{E}-181$} \\
\hline AT1G74910.1 & RVsSFEALQPATR & & & & \\
\hline Os03g11050.3 & RVSSFEALHSATK & & & & \\
\hline D8QZR7 & AMKSPDPLEEQR & \multirow[t]{3}{*}{ Protein kinase superfamily protein } & \multirow[t]{3}{*}{ Protein.postranslational modification.kinase } & \multirow[t]{3}{*}{ OG5_129183 } & \multirow[t]{3}{*}{$1 \mathrm{E}-149$} \\
\hline AT1G67580.1 & MVKSPDPLEEQR & & & & \\
\hline Os02g39010.3 & HMKSPDPLEEQR & & & & \\
\hline D8RF63 & ElsDDEEEEEK & \multirow[t]{3}{*}{ HEAT SHOCK PROTEIN 81.4} & \multirow[t]{3}{*}{ Stress.abiotic.heat } & \multirow[t]{3}{*}{ OG5_126623 } & \multirow[t]{3}{*}{$1 \mathrm{E}-181$} \\
\hline AT5G56000.1 & ElsDDEEEEEK & & & & \\
\hline Os08g39140.1 & ElsDDEDEEEK & & & & \\
\hline D8QX94 & tSCGSPNYAAPEVISGK & \multirow[t]{3}{*}{ SNF1-related protein kinase 1.3} & \multirow[t]{3}{*}{ Protein.postranslational modification } & OG5_126655 & $1 \mathrm{E}-181$ \\
\hline AT5G39440.1 & tSCGSPNYAAPEVISGK & & & & \\
\hline Os03g17980.1 & tSCGSPNYAAPEVISGK & & & & \\
\hline D8SX29 & GEPNISyICSR & SHAGGY-related protein kinase dZeta & Protein.postranslational modification & OG5_126888 & $1 \mathrm{E}-181$ \\
\hline AT2G30980.1 & GEANISyICSR & & & & \\
\hline Os02g14130.1 & GEANISYICSR & & & & \\
\hline D8R4T7 & GGMTsHAAWAR & Pyruvate orthophosphate dikinase & Glycolysis.PPFK & OG5_127082 & $1 \mathrm{E}-181$ \\
\hline AT4G15530.3 & GGMTsHAAWAR & & & & \\
\hline Os05g33570.3 & GGMTSHAAWAR & & & & \\
\hline D8T810 & GLDIDTIQQHYtV & H(+)-ATPase 1 & transport.p- and v-ATPases. $\mathrm{H}+$-exporting & OG5_127253 & $1 \mathrm{E}-181$ \\
\hline AT2G18960.1 & GLDIDTAGHHYtV & & Alpase & & \\
\hline Os04g56160.1 & GLDIDTIQQNYtV & & & & \\
\hline
\end{tabular}


Table 2 Selected conserved phosphorylation sites within the orthologous proteins (Continued)

\begin{tabular}{llll}
\hline D8R651 & VHACVGGtDVR & Eukaryotic translation initiation factor 4A1 & Protein.synthesis.initiation \\
AT3G13920.1 & VHACVGGtSVR & & \\
Medtr2g120800.1 & VHACVGGtSVR & OG5_126984 & \\
D8QVP0 & TIQFVDWCPtGFK & Tubulin alpha-3 & Cell.organisation \\
AT5G19770.1 & TVQFVDWCPtGFK & & OG5_126605 \\
Os03g51600.3 & TIQFVDWCPtGFK & & \\
\hline
\end{tabular}

$\frac{\text { Os03g51600.3 TIQFVDWCPtGFK }}{\text { (1) Phosphorylation sites are indicated as small letters. }}$

Arabidopsis (AT); Rice (Os); Soybean (Glyma); Medicago trunculata (Medtr). 


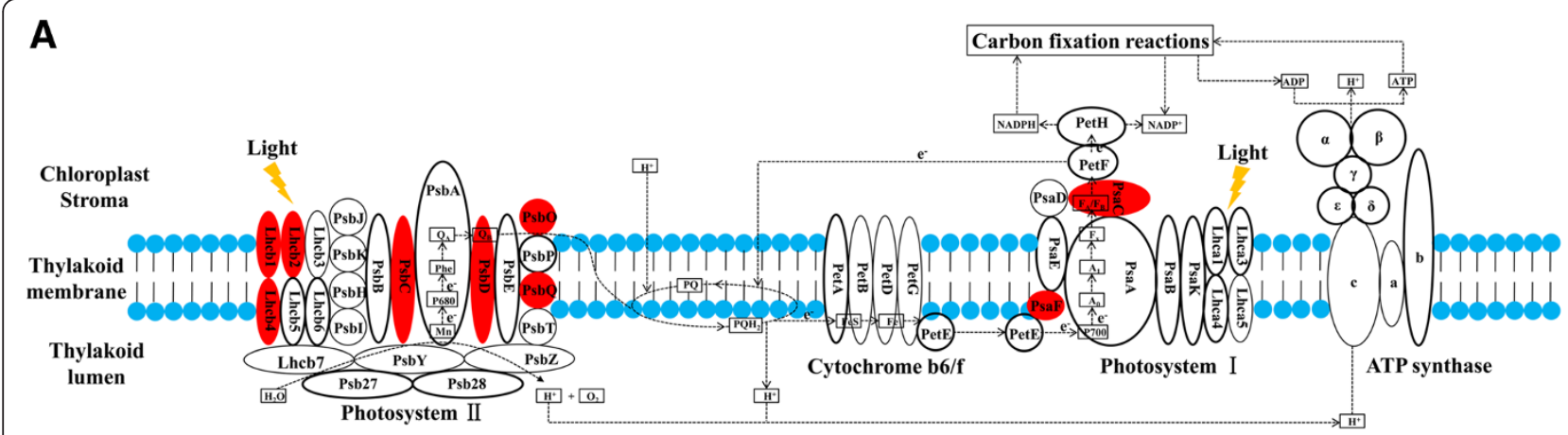

Photosystem II

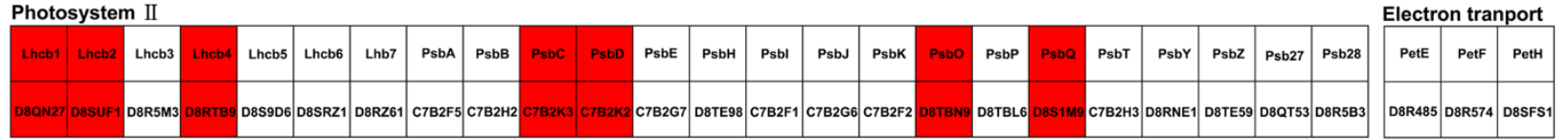

\section{Photosystem I}

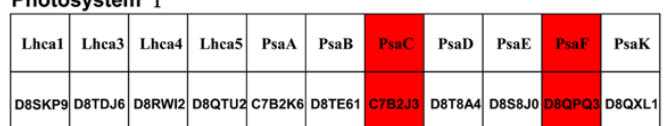

\section{Cytochrome b6/f}

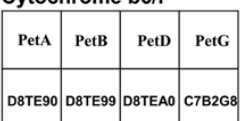

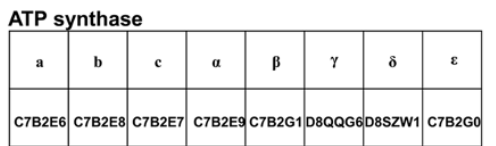

B

Lhcb1

D8QN27_SELML

AT1G29930. 1

0s01g41710.1 ORYSA

A9S6S7_PHYPA

33 ASNGSRIAMRK

27 VLGSGRVTMRK VAKPK-GPSG PWYGSDR 55

21 VFGEGRITMRKSAAKPKPAASGSPWYGADR 50

29 GNVQARVTMR----KASS--SDSIWYGADR 52

Lhcb2

D8SUF1_SELML

AT2G05100. 1

0s03g39610.1 ORYSA

A9S6S7_PHYPA

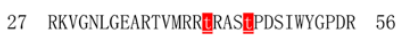

25 RKVGVSGGGRVTMRR VKSTPQSIWYGPDR 54

24 RRVGDSGG-RITMRRTVKSAPQSIWYGPDR 52

26 RKVGNV-QARVTMR-KASSSDSIWYGADR 52

Lhcb4

D8RTB9_SELML

AT3G08940. 2

0s07g37240.1_ORYSA

A9U3M1_PHYPA

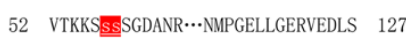

43 KKAK---TVISDR $\cdots$ NL GEVIG R EAVD 115

42 KKAARPSAPTTDR $\cdots$ NNAGEIIGTRFETGE 117

49 KTLKKSSGAGADR $\cdots$ NEAGDVIGTRISKKE 124

PsbD

C7B2K2_SELML.

ATCG00270. 1

0s02g24634.1_0RYSA

1 Mting IVGRLSGGSTSFFDNTDDRPRRDRFAF 30

1 MIIALGKFTKDEKDLFDIMDDWLRRDRFVF 30

PsbC

C7B2К3 SELML

ATCG00280. 1

0s02g24632.1_ORYSA

PSBC_PHYPA
1 MTIALGRVTKEENDLFDIMDDWLRRDRFVF 30

357
Psb0

D8TBN9_SELML

AT3G50820. 1

0s01g31690.1_ORYSA

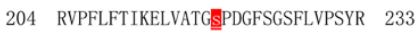

207 RVPFLFTVKQLEASGKPESFSGKFLVPSYR 236

209 RVPFLFTIKNLVATGKPESFGGPFLVPSYR 238

A9RS28_PHYPA 202 RVPFLFTVKELVAEGKPEAFGGSFLVPSYR 231

\section{PsbQ}

D8S1M9_SELML.

AT4G05180. 1

0s07g36080.3_ORYSA

46 DLDLPLKERFFIQPLE

A9S1E8_PHYPA

DFSLALKDRF IQPL P EAAARAKDSAKE 139

36 DTDLPLRERFYLQPLPPAEAAARAKESAQD 65

112 DTDLPLRERFFIQPLSPADAAQRAKFSAQD 141

\section{PsaC}

C7B2J3_SELML

ATCG01060. 1

0s10g21406.1_ORYSA

PSAC_PHYPA

53 RRESACPTDPSSARVYLGSEETTRSTGLAHQ 82

53 RCESACPTDFLSVRVYLWHETTRSMGLAY- 81

53 RCESACPTDFLSVRVYLGPETTRSMALSY- 81

53 RCESACPTDFLSVRVYLGAETTRSMGLAY- 81

PsaF

D8QPQ3_SELML

AT1G31330. 1

0s03g56670.1_ORYSA

A9TWD4_PHYPA

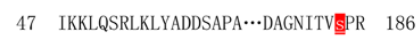

89 IKKLE

100 IKKLQSSLKKYAPDSAPA …DDADIGY--- 236

73 IKKLESRLKLYAPDSAPA $\cdots$ KSGNITVSPR 212

Figure 4 Sellaginella phosphoproteins involved in photosynthesis. (A) Graphical representation of the photosynthesis machineries using the KEGG classification system. Different proteins participating in light-dependent reactions are shown. Circles filled with red color denoted phosphoproteins with confirmed phosphosites identified in this study. UniProtKB accession numbers of Selaginella proteins are shown underneath the corresponding photosynthetic proteins. (B) Alignment of the identified Selaginella photosynthesis phosphoproteins (selected regions) with orthologous sequence from Arabidopsis, rice (ORYSA) and P. patens (PHYPA). Phosphosites identified in this study and in Arabidopsis are highlighted in red and yellow, respectively. Complete alignments of these proteins are available in Additional file 6: Figure S2. Phosphorylation information for the rice and P. patens sequences is not available. 
was identified in maize Lhcb4 [62]. This residue is not conserved in Selaginella Lhcb4 (D8RTB9) but present in Arabidopsis (pThr-109), rice (Thr-111), and moss (Thr-119) (Figure 4B). On the other hand, 2 consecutive phosphosites $($ Ser-57, 58) were detected in D8RTB9and the equivalent Ser residues are only found in moss but not in Arabidopsis or rice. While both of them are located in basic motifs, Ser-58 may also represent a target for acidic casein kinase II.

The PSII core proteins PsbA, PsbD and PsbC are also known to undergo a strong and dynamic redox-regulated phosphorylation cycle [63-65]. STN8-dependent phosphorylation of PSII proteins is required for rapid turnover of photo-damaged PSII complexes and it is highly important during prolonged exposure of the photosynthetic apparatus to excess light [66]. As determined by its structure, STN8 kinase was reported to have a peculiar substrate specificity restricted to the very $\mathrm{N}$-terminal $\mathrm{Thr}$ residue of PsbA, PsbD and PsbC [60]. For example, the phosphosite Thr-2 in Arabidopsis PsbD is phosphorylated by STN8 [42,58]. The same phosphorylation event is detected in Selaginella C7B2K2 (PsbD) while an equivalent Thr residue is found in rice (Figure 4B). On the other hand, while no N-terminal Thr phosphosites were identified in Selaginella C7B2K3 (PsbC), its Thr-346 phosphorylation is conserved in Arabidopsis PsbC [42,52] and equivalent Thr residues are found in rice and moss. This site may represent a substrate of acidic or basic motif recognizing kinases, indicating the possibility of cross-talk between kinases as suggested previously [60].

The oxygen-evolving complex (OEC) is consisted of $\mathrm{PsbO}$, PsbP and PsbQ. PsbO stabilizes the manganese cluster which is the primary site of water splitting. Besides, PsbO regulates dephosphorylation and turnover of the PSII reaction center PsbA $[67,68]$. However, no phosphorylation events in PsbO have been reported previously in any plants. In Selaginella D8TBN9 (PsbO), we identified a unique Pro-directed Ser-219 phosphosite. The equivalent residues in other PsbO sequences examined are all Lys which is non-phosphorylatable (Figure 4B). PsbQ is required for PSII assembly, stability, and photoautotrophic growth under low light conditions [69]. The Selaginella PsbQ (D8S1M9) was found to be phosphorylated at the Ser-61 residue, which is a potential target of Prodirected kinase. Equivalent pSer and Ser residues are found in Arabidopsis and moss PsbQ sequences, respectively (Figure 4B).

PsaC and PsaF are components of PSI which performs the light-induced electron transfer from plastocyanin or cytochrome c6 (Cytc) to ferredoxin. As a chloroplastencoded PSI subunit, PsaC binds the two terminal electron acceptors $\left(\mathrm{F}_{\mathrm{A}}\right.$ and $\left.\mathrm{F}_{\mathrm{B}}\right)$. No phosphorylation was reported in $\mathrm{PsaC}$ previously in any plants. $\mathrm{PsaC}$ is extremely conserved among the four plant species examined here with most of the residues identical (Additional file 6: Figure S2). Intriguingly, the phosphorylation event occurs at a unique residue (Ser-71) in Selaginella PsaC (C7B2J3). The equivalent residues in the other plant sequences are all non-phosphorylatable. The nuclear subunit PsaF provides a docking site for plastocyanin and Cytc on the lumenal side of PSI. In Arabidopsis, PsaF was reported to be phosphorylated at Ser-94, Ser-95, Tyr-99, and Ser-103 $[42,52]$. Most of the equivalent residues in Selaginella PsaF (D8QPQ3) are conserved except for Ser-95. On the other hand, the Ser-184 phosphosite in D8QPQ3 is located in the very $\mathrm{C}$-terminal region which is absent in Arabidopsis and rice. The same residue was identified in the moss PsaF sequence, suggesting that the Ser-184 phosphorylation event might have been lost during the evolution of flowering plants.

Overall, several phosphorylated residues in the Selaginella photosynthesis proteins are conserved with equivalent phosphorylation in Arabidopsis and/or phosphorylatable residues in most of the plants examined, including Lhcb1: Thr-44, Lhcb2: Thr-42 and 46,PsbD: Thr-2,PsbC: Thr-346, and psbQ: Ser-61. The phosphorylation of Thr-46 in Lhcb2 is first identified in Selaginella and the equivalent residues in other plant sequences are likely to be phosphorylated. We also identified unique phosphorylated residues within highly conserved regions in Selaginella PsbO (Ser-219) and PsaC (Ser-71). On the other hand, phosphorylation events with equivalent residues only in moss were detected in Selaginella Lhcb1, Lhbc4 and PsaF. These phosphosites are located in low-homology regions when compared with the Arabidopsis and rice sequences, implicating that they were lost in the flowering plants during evolution. It will be very interesting to investigate how the different unique phosphorylation events are involved in light reactions in Selaginella.

\section{Conclusions}

Our work generates the first large-scale atlas of phosphoproteins in Selaginella which occupies a unique position in the evolution of terrestrial plants. Combining PEG fractionation with IMAC enrichment, a total of 1593 unique phosphopeptides (1588 individual phosphosites) representing 851 unique phosphoproteins were retrieved. An overview of the Selaginella phosphoproteomics data revealed general features which are largely consistent with the dicot model Arabidopsis. Known plant phosphorylation Ser/Thr motifs were extracted from total and Selaginella-specific phosphopeptides, implicating the conservation of phosphorylation machineries during vascular plant evolution. In fact, 97highly conserved phosphorylation events were identified among Selaginella and flowering plant homologs. In PSI proteins, we identified conserved residues which are potential targets of STN7 and STN8 kinases. On the other hand, several phosphosites unique to 
Selaginella were detected in the highly conserved PSI and PSII proteins. Future research into functional roles of Selaginella-specific phosphorylation events in photosynthesis and other processes may offer insight into the molecular mechanisms leading to the distinct evolution of lycophytes.

\section{Methods}

\section{Protein extraction and PEG fractionation}

Two-gram aerial tissues of wild-growing Selaginella moellendorffii collected from the Victoria Peak in Hong Kong were ground to fine powder in liquid nitrogen. The powder was homogenized in $10 \mathrm{~mL}$ of ice-cold Mg/NP-40 extraction buffer containing $0.5 \mathrm{M}$ Tris- $\mathrm{HCl}$ (pH 8.3), $20 \mathrm{mM} \mathrm{MgCl}_{2}, 2 \% \mathrm{v} / \mathrm{v} \mathrm{NP}-40,2 \% \mathrm{v} / \mathrm{v} \beta$-mercaptoethanol, $1 \mathrm{mM}$ phenylmethylsulfonyl fluoride and $1 \% \mathrm{w} / \mathrm{v}$ polyvinylpolypyrrolidone using the Tissue-Tearor (BioSpec) operated at maximum speed for $1 \mathrm{~min}$ on ice [70]. After centrifugation at $12000 \times \mathrm{g}$ for $15 \mathrm{~min}$ at $4^{\circ} \mathrm{C}$, the supernatant was treated with 15\% PEG-4000 and incubated on ice for $30 \mathrm{~min}$, followed by centrifugation at $1500 \times \mathrm{g}$ for $10 \mathrm{~min}$ at $4^{\circ} \mathrm{C}$. The pellet was washed sequentially with ice-cold 10\% trichloroacetic acid/acetone, ice-cold 100\% methanol containing $0.1 \mathrm{M}$ ammonium acetate, and icecold $100 \%$ acetone. The supernatant was precipitated by adding four volumes of ice-cold acetone and then incubated at $-20^{\circ} \mathrm{C}$ for $2 \mathrm{~h}$. After centrifugation at $12000 \times \mathrm{g}$ for $5 \mathrm{~min}$ at $4^{\circ} \mathrm{C}$, the pellet was rinsed as described above. For the plant debris left after the initial Mg/NP-40 extraction, residual protein was extracted by $4 \%$ SDS. After centrifugation, the supernatant was precipitated with ice-cold acetone, followed by sequential rinsing of the pellet.

\section{Protein digestion and phosphopeptide enrichment}

The pellets obtained from each of the above step were re-suspended in solution containing $0.2 \mathrm{M}$ Tris- $\mathrm{HCl}$ ( $\mathrm{pH}$ 8.0), $8 \mathrm{M}$ urea and $4 \mathrm{mM} \mathrm{CaCl}$. Dissolved protein samples were reduced with $10 \mathrm{mM}$ dithiothreitol for $30 \mathrm{~min}$ at $56^{\circ} \mathrm{C}$, and the alkylated with $40 \mathrm{mM}$ iodoacetamide for $30 \mathrm{~min}$ at room temperature in the dark. Protein concentration was measured by the Bio-Rad Protein Assay kit. Afterwards, trypsin (Worthington) was added in a 1:50 (enzyme: protein) $\mathrm{w} / \mathrm{w}$ ratio and the mixture incubated overnight at $37^{\circ} \mathrm{C}$. Trypsinized peptides were loaded onto a 1 g Sep-Pak C18 column (Waters), washed twice with $10 \mathrm{~mL} \mathrm{1 \%}$ acetic acid, eluted with $7 \mathrm{~mL} \mathrm{80 \%} \mathrm{acetonitrile}$ containing $0.1 \%$ acetic acid, dried under speed-vacuum, re-suspended in $400 \mu \mathrm{L} 1 \%$ acetic acid, and then loaded onto a mini-column of $40 \mu \mathrm{L}$ IMAC resin prepared as described previously [71]. The IMAC mini-column was rinsed twice with $40 \mu \mathrm{L}$ wash buffer containing $25 \% \mathrm{v} / \mathrm{v}$ acetonitrile, $100 \mathrm{mM} \mathrm{NaCl}$ and $0.1 \% \mathrm{v} / \mathrm{v}$ acetic acid, then washed once each with $40 \mu \mathrm{L} 1 \% \mathrm{v} / \mathrm{v}$ acetic acid and $20 \mu \mathrm{L}$ double-distilled water, eluted with $120 \mu \mathrm{L} 6 \% \mathrm{w} / \mathrm{v} \mathrm{NH}_{3}$.
$\mathrm{H}_{2} \mathrm{O}$, and dried under speed-vacuum. IMAC-enriched phosphopeptides derived from different PEG fractionated samples (Additional file 1: Figure S1) were subject to LCMS/MS analysis.

\section{RPLC-ESI-MS/MS detection}

The Triple TOF 5600 mass spectrometer (AB SCIEX), a hybrid quadrupole TOF platform [72], was coupled with an Nano-LC system (Agilent) utilizing Nanospray III ion-source (AB SCIEX). Mobile phase A ( $2 \% \mathrm{ACN}, 0.1 \%$ formic acid) and mobile phase B (98\% ACN, 0.1\% formic acid) were used to establish a 120 min gradient comprised of $80 \mathrm{~min}(5-30 \% \mathrm{~B}), 12 \mathrm{~min}(30-60 \% \mathrm{~B}), 6 \mathrm{~min}$ (60-90\% B), 10 min (90\% B), and $12 \min (90-5 \%$ B). The flow rate was $300 \mathrm{~nL} / \mathrm{min}$. Peptides were separated on a fused silica capillary emitter (New Objective) packed inhouse with $5 \mu \mathrm{m} \mathrm{C18}$ resin (New Objective), and analyzed in positive ion mode by electrospray ionization. For information dependent acquisition, each survey scan was acquired in $250 \mathrm{~ms}$ followed by 20 product ion scans collected in $50 \mathrm{~ms} /$ per scan.

\section{Database searching of MS/MS spectra}

For proteome analysis, raw data from Triple TOF 5600 were searched with ProteinPilot software (version 4.0, AB SCIEX) against the Uniprot Selaginella moellendorffii complete proteome database (downloaded in April 2011, 33195 sequences) using following parameters: Sample Type (Identification), Cys Alkylation (Iodoacetamide), Digestion (Trypsin), Search Effort (Rapid). The false discovery rate (FDR) analysis was done by using the tool integrated in ProteinPilot. All data were filtered at 1\% FDR.

For phosphoproteome analysis, raw data MS/MS (wiff files) were converted to .mgf files and searched with the Mascot (version 2.2, Matrix Science) software [73] against the Selaginella proteome database using following parameters: fixed modifications was set to carbamidomethylation on cysteine, variable modifications was set to oxidation of methionine and phosphorylation at serine, threonine and tyrosine, peptide and MS/MS fragment tolerances were set to $20 \mathrm{ppm}$ and $0.2 \mathrm{Da}$ respectively, trypsin was selected as digestion enzyme, and up to two missed cleavages were allowed. All .mgf files were merged into one file followed by database searching.

\section{Post-search data processing and phosphosite localization}

The Mascot search result was first loaded into Scaffold (version 3.0, Proteome Software) for further analysis. In order to screen phosphopeptides with high confidence, "Min Protein" (protein identification probability), "Min \# Peptide" (the number of unique peptides on which a protein identification is based) and "Min Peptide" (peptide identification probability) were adjusted to $20 \%, 1$ and 95\% respectively $[74,75]$. Afterwards, the mzIdentML file 
generated by Scaffold was loaded into Scaffold PTM (version 1.1, Proteome Software) to determine the localization probability of phosphosites [76].

\section{Gene ontology annotations}

Gene ontology (GO) annotations of all identified proteins and phosphoproteins in Selaginella categorized into 3 classifications (Cellular Component, Molecular Function and Biological Process) were batch-retrieved from the Protein Information Resource (http://pir.georgetown. edu/pirwww/search/batch.shtml).

\section{Analysis of phosphorylation site conservation}

The Selaginella phosphopeptides were clustered with different plant phosphopeptides retrieved from the Plant Protein Phosphorylation Database $\left(\mathrm{P}^{3} \mathrm{DB}\right.$; http://www. p3db.org/) using the CD-HIT web server [54] (http:// www.bioinformatics.org/cd-hit/). All phosphopeptide sequences were combined into a single Fasta file for data upload. Default parameters were adopted together with a $60 \%$ similarity cutoff and a bandwidth of 5 . Conservation of phosphorylation sites among different plant species were then identified by manual inspection of the sequence alignment in each cluster.

\section{Phosphorylation motif analysis}

Sequence was centered on each phosphosite and extended to 15 amino acids ( \pm 7 residues). Phosphosites, which could not be extended because of $\mathrm{N}$ - or C-termini, were excluded from motif analysis. Only phosphosites with localization probability above 95\% were used. General phosphorylation motif classes were assigned as defined previously [50]: $\mathrm{P}$ at +1 (Pro-directed); $\mathrm{D} / \mathrm{E}$ at $+1 /+2$ or +3 (Acidic), 5 or more $\mathrm{D} / \mathrm{E}$ at +1 to +6 (Acidic); $\mathrm{K} / \mathrm{R}$ at -3 (Basic), 2 or more $\mathrm{K} / \mathrm{R}$ at -6 to -1 (Basic); otherwise (Others). Specific motifs were extracted from the data set by using motif-x algorithm (http://motif-x.med.harvard. edu/motif-x.html) [53]. The Selaginella proteome database in fasta format was retrieved (http://www.phytozome. $\mathrm{com} /$ ) and uploaded as background. The significance threshold was set to $10^{-6}$ and the minimum number of motif occurrences was 20 .

\section{Additional files}

Additional file 1: Figure S1. SDS-PAGE analysis of protein samples after PEG fractionation. Total protein (T) was obtained from the supernatant after extraction of tissues in Mg/NP-buffer. Residual protein (R) was obtained by extracting the plant debris in 4\% SDS. The supernatant was subject to $15 \%$ PEG precipitation and the pellet $(P)$ was resuspended in urea-containing buffer. Protein in the final supernatant (S) was precipitated by acetone and resuspended in urea-containing buffer. Each lane was loaded with $50 \mu \mathrm{g}$ of protein. Following Coomassie blue staining, the large subunit of RUBISCO (RBCL) can be visualized as a discrete band corresponding to a MW of $\sim 55 \mathrm{kDa}$. Note the substantially reduced abundance of $\mathrm{RBCL}$ in the sample (S) after PEG precipitation.
The fractionated samples (, , P, and R) were trypsin-digested, followed by IMAC enrichment of phosphopeptides.

Additional file 2: Table S1. List of all identified Selaginella phosphopeptides. Additional file 3: Table S2. List of 716 Selaginella phosphoproteins (localization probability $\geq 95 \%$ ) with OrthoMCL Group information.

Additional file 4: Table S3. List of Selaginella phosphopeptides with the KXXS motif.

Additional file 5: Table S4. List of Selaginella phosphopeptides with conserved phosphosites in other plant phosphopeptides.

Additional file 6: Figure S2. Complete ClustalW sequence alignment of the nine identified Selaginella PSI proteins with Arabidopsis, rice and moss orthologous sequences. Phosphosites identified in this study and in Arabidopsis are highlighted in red and yellow, respectively. The rice and moss sequences are included as references although no phosphorylation information is available for these proteins.

\section{Abbreviations}

FDR: False discovery rate; GO: Gene ontology; IMAC: Immobilized metal affinity chromatography; $P^{3} \mathrm{DB}$ : Plant protein phosphorylation database; PEG: Polyethylene glycol; PSI: Photosystem I; PSII: Photosystem II; PTMs: Posttranslational modifications; RUBISCO: Ribulose-1,5-bisphosphate carboxylase/ oxygenase.

\section{Competing interests}

The authors declare that they have no competing interests.

\section{Authors' contributions}

$X C, W L C$, and $C L$ initially conceived of and designed the proteomics experiments. XC and WLC participated in all experimental procedures, data analysis, and manuscript preparation. FYZ was involved in data analysis. CL finalized the manuscript for submission. All authors read and approved the final manuscript.

\section{Acknowledgements}

This work is supported by the HKU Seed Funding Programme for Basic Research (201011159033) and HKU Small Project Funding (201209176039).

\section{Author details}

${ }^{1}$ School of Biological Sciences, The University of Hong Kong, Pokfulam Hong Kong, China. 'Wuhan Institute of Biotechnology, Wuhan, Hubei, China.

Received: 31 December 2013 Accepted: 6 March 2014

Published: 17 March 2014

\section{References}

1. Kenrick P, Crane PR: The origin and early evolution of plants on land. Nature 1997, 389:33-39.

2. Banks JA, Nishiyama T, Hasebe M, Bowman JL, Gribskov M, dePamphilis C, Albert VA, Aono N, Aoyama T, Ambrose BA, Ashton NW, Axtell MJ, Barker E, Barker MS, Bennetzen JL, Bonawitz ND, Chapple C, Cheng C, Correa LG, Dacre M, DeBarry J, Dreyer I, Elias M, Engstrom EM, Estelle M, Feng L, Finet C, Floyd SK, Frommer WB, Fujita T, et al: The Selaginella genome identifies genetic changes associated with the evolution of vascular plants. Science 2011, 332:960-963.

3. Wang W, Tanurdzic M, Luo M, Sisneros N, Kim HR, Weng JK, Kudrna D, Mueller C, Arumuganathan K, Carlson J, Chapple C, de Pamphilis C, Mandoli D, Tomkins J, Wing RA, Banks JA: Construction of a bacterial artificial chromosome library from the spikemoss Selaginella moellendorffii: a new resource for plant comparative genomics. BMC Plant Biol 2005, 5:10.

4. Banks JA: Selaginella and 400 million years of separation. Annu Rev Plant Biol 2009, 60:223-238.

5. Yin C, Richter U, Borner T, Weihe A: Evolution of phage-type RNA polymerases in higher plants: characterization of the single phage-type RNA polymerase gene from Selaginella moellendorffii. J Mol Evol 2009, 68:528-538.

6. Hedman H, Kallman T, Lagercrantz U: Early evolution of the MFT-like gene family in plants. Plant Mol Biol 2009, 70:359-369.

7. Alvarez-Venegas R, Avramova Z: Evolution of the PWWP-domain encoding genes in the plant and animal lineages. BMC Evol Biol 2012, 12:101. 
8. Tegeder M, Ward JM: Molecular evolution of plant AAP and LHT amino acid transporters. Front Plant Sci 2012, 3:21.

9. Gomez-Porras JL, Riano-Pachon DM, Benito B, Haro R, Sklodowski K, Rodriguez-Navarro A, Dreyer I: Phylogenetic analysis of $k(+)$ transporters in bryophytes, lycophytes, and flowering plants indicates a specialization of vascular plants. Front Plant Sci 2012, 3:167.

10. Pedersen CN, Axelsen KB, Harper JF, Palmgren MG: Evolution of plant p-type ATPases. Front Plant Sci 2012, 3:31

11. Kopriva S, Wiedemann G, Reski R: Sulfate assimilation in basal land plants what does genomic sequencing tell us? Plant Biol (Stuttg) 2007, 9:556-564.

12. Hirano K, Nakajima M, Asano K, Nishiyama T, Sakakibara H, Kojima M, Katoh E, Xiang H, Tanahashi T, Hasebe M, Banks JA, Ashikari M, Kitano H, Ueguchi-Tanaka M, Matsuoka M: The GID1-mediated gibberellin perception mechanism is conserved in the lycophyte Selaginella moellendorffii but not in the bryophyte Physcomitrella patens. Plant Cell 2007, 19:3058-3079.

13. Paponov IA, Teale W, Lang D, Paponov M, Reski R, Rensing SA, Palme K: The evolution of nuclear auxin signalling. BMC Evol Biol 2009, 9:126.

14. Rychel $A L$, Peterson KM, Torii KU: Plant twitter: ligands under 140 amino acids enforcing stomatal patterning. J Plant Res 2010, 123:275-280.

15. Eklund DM, Svensson EM, Kost B: Physcomitrella patens: a model to investigate the role of RAC/ROP GTPase signalling in tip growth. J Exp Bot 2010, 61:1917-1937.

16. Hanada K, Hase T, Toyoda T, Shinozaki K, Okamoto M: Origin and evolution of genes related to $A B A$ metabolism and its signaling pathways. J Plant Res 2011, 124:455-465.

17. Smith DR: Unparalleled GC content in the plastid DNA of Selaginella. Plant Mol Biol 2009, 71:627-639.

18. Shakirov EV, Shippen DE: Selaginella moellendorffii telomeres: conserved and unique features in an ancient land plant lineage. Front Plant Sci 2012, 3:161.

19. Chan AP, Melake-Berhan A, O'Brien K, Buckley S, Quan H, Chen D, Lewis M, Banks JA, Rabinowicz PD: The highest-copy repeats are methylated in the small genome of the early divergent vascular plant Selaginella moellendorffii. BMC Genomics 2008, 9:282.

20. Axtell MJ, Snyder JA, Bartel DP: Common functions for diverse small RNAs of land plants. Plant Cell 2007, 19:1750-1769.

21. Hecht J, Grewe F, Knoop V: Extreme RNA editing in coding islands and abundant microsatellites in repeat sequences of Selaginella moellendorffii mitochondria: the root of frequent plant mtDNA recombination in early tracheophytes. Genome Biol Evol 2011, 3:344-358.

22. Novikova O, Smyshlyaev G, Blinov A: Evolutionary genomics revealed interkingdom distribution of Tcn1-like chromodomain-containing Gypsy LTR retrotransposons among fungi and plants. BMC Genomics 2010, 11:231.

23. Wang $Y H$, Long $C L$, Yang FM, Wang $X$, Sun QY, Wang HS, Shi $Y N$, Tang GH: Pyrrolidinoindoline alkaloids from Selaginella moellendorfii. J Nat Prod 2009, 72:1151-1154.

24. Wu B, Wang J: Phenolic compounds from Selaginella moellendorfii. Chem Biodivers 2011, 8:1735-1747.

25. Cao Y, Tan NH, Chen JJ, Zeng GZ, Ma YB, Wu YP, Yan H, Yang J, Lu LF, Wang Q: Bioactive flavones and biflavones from Selaginella moellendorffii Hieron. Fitoterapia 2010, 81:253-258.

26. Wang HS, Sun L, Wang YH, Shi YN, Tang GH, Zhao FW, Niu HM, Long CL, $\mathrm{Li}$ L: Carboxymethyl flavonoids and a monoterpene glucoside from Selaginella moellendorffii. Arch Pharm Res 2011, 34:1283-1288.

27. Wang X, Chen S, Zhang H, Shi L, Cao F, Guo L, Xie Y, Wang T, Yan X, Dai S: Desiccation tolerance mechanism in resurrection fern-ally Selaginella tamariscina revealed by physiological and proteomic analysis. J Proteome Res 2010, 9:6561-6577.

28. Muir T: Posttranslational modification of proteins: expanding nature's inventory: by Christopher T Walsh. Chem Bio Chem 2006, 7:1623-1624.

29. Hubbard MJ, Cohen P: On target with a new mechanism for the regulation of protein phosphorylation. Trends Biochem Sci 1993, 18:172-177.

30. Zolnierowicz S, Bollen M: Protein phosphorylation and protein phosphatases: De Panne, Belgium, September 19-24, 1999. EMBO J 2000, 19:483-488

31. De la Fuente van Bentem S, Hirt H: Using phosphoproteomics to reveal signalling dynamics in plants. Trends Plant Sci 2007, 12:404-411.
32. Initiative TAG: Analysis of the genome sequence of the flowering plant Arabidopsis thaliana. Nature 2000, 408:796-815.

33. Manning G, Whyte DB, Martinez R, Hunter $T$, Sudarsanam $S$ : The protein kinase complement of the human genome. Science 2002, 298:1912-1934.

34. Stone JM, Walker JC: Plant protein kinase families and signal transduction. Plant Physiol 1995, 108:451-457.

35. Des Francs CC, Thiellement $H$, De Vienne D: Analysis of leaf proteins by two-dimensional gel electrophoresis: protease action as exemplified by ribulose bisphosphate carboxylase/oxygenase degradation and procedure to avoid proteolysis during extraction. Plant Physiol 1985, 78:178-182.

36. Aryal UK, Krochko JE, Ross AR: Identification of phosphoproteins in Arabidopsis thaliana leaves using polyethylene glycol fractionation, immobilized metal-ion affinity chromatography, two-dimensional gel electrophoresis and mass spectrometry. J Proteome Res 2012, 11:425-437

37. Xi J, Wang X, Li S, Zhou X, Yue L, Fan J, Hao D: Polyethylene glycol fractionation improved detection of low-abundant proteins by twodimensional electrophoresis analysis of plant proteome. Phytochemistry 2006, 67:2341-2348.

38. Lee $D G$, Ahsan $N$, Lee $S H$, Kang KY, Lee JJ, Lee $B H$ : An approach to identify cold-induced low-abundant proteins in rice leaf. C R Biol 2007 330:215-225.

39. Acquadro A, Falvo S, Mila S, Giuliano Albo A, Comino C, Moglia A Lanteri S: Proteomics in globe artichoke: protein extraction and sample complexity reduction by PEG fractionation. Electrophoresis 2009, 30:1594-1602.

40. Scanff P, Yvon M, Pelissier JP: Immobilized Fe3+ affinity chromatographic isolation of phosphopeptides. J Chromatogr 1991, 539:425-432.

41. Benschop JJ, Mohammed S, O'Flaherty M, Heck AJ, Slijper M, Menke FL: Quantitative phosphoproteomics of early elicitor signaling in Arabidopsis. Mol Cell Proteomics 2007, 6:1198-1214.

42. Reiland S, Messerli G, Baerenfaller K, Gerrits B, Endler A, Grossmann J, Gruissem W, Baginsky S: Large-scale Arabidopsis phosphoproteome profiling reveals novel chloroplast kinase substrates and phosphorylation networks. Plant Physiol 2009, 150:889-903.

43. Nakagami H, Sugiyama N, Mochida K, Daudi A, Yoshida Y, Toyoda T, Tomita M, Ishihama $Y$, Shirasu K: Large-scale comparative phosphoproteomics identifies conserved phosphorylation sites in plants. Plant Physio/ 2010 153:1161-1174.

44. Meyer LJ, Gao J, Xu D, Thelen JJ: Phosphoproteomic analysis of seed maturation in Arabidopsis, rapeseed, and soybean. Plant Physiol 2012, 159:517-528.

45. Grimsrud PA, den Os D, Wenger CD, Swaney DL, Schwartz D, Sussman MR, Ane JM, Coon JJ: Large-scale phosphoprotein analysis in Medicago truncatula roots provides insight into in vivo kinase activity in legumes. Plant Physiol 2010, 152:19-28.

46. Bi YD, Wang HX, Lu TC, Li XH, Shen Z, Chen YB, Wang BC: Large-scale analysis of phosphorylated proteins in maize leaf. Planta 2011, 233:383-392.

47. Rudrabhatla P, Reddy MM, Rajasekharan R: Genome-wide analysis and experimentation of plant serine/threonine/tyrosine-specific protein kinases. Plant Mol Biol 2006, 60:293-319.

48. Li L, Stoeckert CJ Jr, Roos DS: OrthoMCL: identification of ortholog groups for eukaryotic genomes. Genome Res 2003, 13:2178-2189.

49. Chen F, Mackey AJ, Vermunt JK, Roos DS: Assessing performance of orthology detection strategies applied to eukaryotic genomes. PLoS One 2007, 2:e383.

50. Villen J, Beausoleil SA, Gerber SA, Gygi SP: Large-scale phosphorylation analysis of mouse liver. Proc Natl Acad Sci U S A 2007, 104:1488-1493.

51. Nuhse TS, Stensballe A, Jensen ON, Peck SC: Phosphoproteomics of the Arabidopsis plasma membrane and a new phosphorylation site database. Plant Cell 2004, 16:2394-2405.

52. Sugiyama N, Nakagami H, Mochida K, Daudi A, Tomita M, Shirasu K, Ishihama Y: Large-scale phosphorylation mapping reveals the extent of tyrosine phosphorylation in Arabidopsis. Mol Syst Biol 2008, 4:193.

53. Schwartz D, Gygi SP: An iterative statistical approach to the identification of protein phosphorylation motifs from large-scale data sets. Nat Biotechnol 2005, 23:1391-1398.

54. Li W, Godzik A: Cd-hit: a fast program for clustering and comparing large sets of protein or nucleotide sequences. Bioinformatics 2006, 22:1658-1659. 
55. Jansson S: The light-harvesting chlorophyll a/b-binding proteins. Biochim Biophys Acta 1994, 1184:1-19.

56. Bellafiore S, Barneche F, Peltier G, Rochaix JD: State transitions and light adaptation require chloroplast thylakoid protein kinase STN7. Nature 2005, 433:892-895.

57. Bennett J: Chloroplast phosphoproteins: phosphorylation of polypeptides of the light-harvesting chlorophyll protein complex. Eur J Biochem 1979, 99:133-137.

58. Vener AV, Harms A, Sussman MR, Vierstra RD: Mass spectrometric resolution of reversible protein phosphorylation in photosynthetic membranes of Arabidopsis thaliana. J Biol Chem 2001, 276:6959-6966.

59. Tikkanen M, Piippo M, Suorsa M, Sirpio S, Mulo P, Vainonen J, Vener AV, Allahverdiyeva Y, Aro EM: State transitions revisited-a buffering system for dynamic low light acclimation of Arabidopsis. Plant Mol Biol 2006, 62:779-793.

60. Vainonen JP, Hansson M, Vener AV: STN8 protein kinase in Arabidopsis thaliana is specific in phosphorylation of photosystem II core proteins. $J$ Biol Chem 2005, 280:33679-33686.

61. Bergantino E, Dainese P, Cerovic Z, Sechi S, Bassi R: A post-translational modification of the photosystem II subunit CP29 protects maize from cold stress. J Biol Chem 1995, 270:8474-8481.

62. Bergantino E, Sandona D, Cugini D, Bassi R: The photosystem II subunit CP29 can be phosphorylated in both C3 and C4 plants as suggested by sequence analysis. Plant Mol Biol 1998, 36:11-22.

63. Aro EM, Ohad I: Redox regulation of thylakoid protein phosphorylation. Antioxid Redox Signal 2003, 5:55-67.

64. Vener AV: Environmentally modulated phosphorylation and dynamics of proteins in photosynthetic membranes. Biochim Biophys Acta 2007 1767:449-457.

65. Rochaix JD: Role of thylakoid protein kinases in photosynthetic acclimation. FEBS Lett 2007, 581:2768-2775.

66. Tikkanen M, Nurmi M, Kangasjarvi S, Aro EM: Core protein phosphorylation facilitates the repair of photodamaged photosystem II at high light. Biochim Biophys Acta 2008, 1777:1432-1437.

67. Yi X, McChargue M, Laborde S, Frankel LK, Bricker TM: The manganesestabilizing protein is required for photosystem II assembly/stability and photoautotrophy in higher plants. J Biol Chem 2005, 280:16170-16174.

68. Lundin B, Hansson M, Schoefs B, Vener AV, Spetea C: The Arabidopsis PsbO2 protein regulates dephosphorylation and turnover of the photosystem II reaction centre D1 protein. Plant J 2007, 49:528-539.

69. Yi X, Hargett SR, Frankel LK, Bricker TM: The PsbQ protein is required in Arabidopsis for photosystem II assembly/stability and photoautotrophy under low light conditions. J Biol Chem 2006, 281:26260-26267.

70. Kim ST, Cho KS, Jang YS, Kang KY: Two-dimensional electrophoretic analysis of rice proteins by polyethylene glycol fractionation for protein arrays. Electrophoresis 2001, 22:2103-2109.

71. Chen X, WU D, Zhao Y, Wong BH, Guo L: Increasing phosphoproteome coverage and identification of phosphorylation motifs through combination of different HPLC fractionation methods. J Chromatogr B Analyt Technol Biomed Life Sci 2011, 879:25-34

72. Andrews GL, Simons BL, Young JB, Hawkridge AM, Muddiman DC Performance characteristics of a new hybrid quadrupole time-of-flight tandem mass spectrometer (TripleTOF 5600). Anal Chem 2011, 83:5442-5446.

73. Perkins DN, Pappin DJ, Creasy DM, Cottrell JS: Probability-based protein identification by searching sequence databases using mass spectrometry data. Electrophoresis 1999, 20:3551-3567.

74. Keller A, Nesvizhskii Al, Kolker E, Aebersold R: Empirical statistical model to estimate the accuracy of peptide identifications made by MS/MS and database search. Anal Chem 2002, 74:5383-5392.

75. Nesvizhskii Al, Keller A, Kolker E, Aebersold R: A statistical model for identifying proteins by tandem mass spectrometry. Anal Chem 2003, 75:4646-4658.

76. Beausoleil SA, Villen J, Gerber SA, Rush J, Gygi SP: A probability-based approach for high-throughput protein phosphorylation analysis and site localization. Nat Biotechnol 2006, 24:1285-1292.

doi:10.1186/1477-5956-12-16

Cite this article as: Chen et al:: Phosphoproteomic analysis of the non-seed vascular plant model Selaginella moellendorffii. Proteome Science 2014 12:16.

\section{Submit your next manuscript to BioMed Central and take full advantage of:}

- Convenient online submission

- Thorough peer review

- No space constraints or color figure charges

- Immediate publication on acceptance

- Inclusion in PubMed, CAS, Scopus and Google Scholar

- Research which is freely available for redistribution 